\title{
REMARKS ON THE DIFFERENTIAL FORMS OF THE FIRST KIND ON ALGEBRAIC VARIETIES
}

\author{
YÛSAKU KAWAHARA
}

$\$ 1$. A differential form $\omega$ on a complete variety $\mathbf{U}^{\prime \prime}$ is said to be of the first kind if it is finite at every simple point of any variety which is birationally equivalent to $\mathbf{U}$. Let $k$ be a common field of definition for $\mathbf{U}$ and $\omega$, and let $\mathbf{P}$ be a generic point of $\mathbf{U}$ over $k$. If $\omega$ is of the first kind, then $\omega(\mathbf{P})$ is of course a differential form of the first kind belonging to the extension $k(\mathbf{P})$ of $k$. With respect to the converse, we prove the following

THEOREM 1. Let $k$ be a field of definition for a complete variety $\mathbf{U}^{n}$ and a aifferential form $\omega$ on $\mathbf{U}$, and let $\mathbf{P}$ be a generic point of $\mathbf{U}$ over $k$. Let $k$ be a perfect ${ }^{11}$ field or more generally let $k$ have a perfect ${ }^{1)}$ subficld which is a field of definition for $\mathbf{U}$. If $\omega(\mathbf{P})$ is a differential form of the first kind belonging to the extension $k(\mathbf{P})$ of $k$, then $\omega$ is of the first kind. ${ }^{2}$

Proof. Let $V$ be a variety which is birationally equivalent to $\mathbf{U}$ and let $K$ be a field of definition of the birational correspondence between $U$ and $V$. We may assume without loss of generality that $K$ is algebraically closed and contains $k$ and that $\mathbf{P}$ is a generic point of $\mathbf{U}$ over $K$. We want to show that (1) is finite at every simple point of $V$. It suffices to show that $\omega(\mathbf{P})$, considered as the difterential form belonging to the extension $K(\mathbf{P})$ of $K$, is of the first kind or that $\omega(\mathbf{P})$ is finite at every prime divisor $\mathfrak{P}$ in the sense of Zariski of $K(\mathbf{P})$ ( = valuation of $K(\mathbf{P})$ of dimension $n-1$ over $K$ ), namely, that $\omega(\mathbf{P}$ ) is of the form

$$
\omega(\mathbf{P})=\sum z_{\alpha_{3}^{3}} \ldots d y_{\alpha} d y_{3} \ldots ;
$$

$z_{\alpha \beta} \ldots, y_{\alpha}, y_{3}$, etc. being in the valuation ring of $\mathfrak{P}^{31}$

We first prove

Lemma. Lei $K$ be a ficld, $k$ a subfield of $K$; let $(x)$ be a sel of quantities, such that $K$ and $k(x)$ are independent over $k$. Then if $v$ is a valuation of $K(x)$

Received April 22, 1953.

1) If we omit the condition of perfectness this theorem does not hold in general.

2) If the problem of the reduction of the singularity over perfect field is solved affirmatively, this theorem is an immediate consequence of theorem 1 of $\mathrm{S}$. Koizumi's paper; On the differential forms of the first kind on algebraic varieties, Journal of the Mathematical Society of Japan, Vol. 2. However it would not be meaningless to give a simple direct proof.

2. See Y. Kawahara, On the differential forms on algebraic varieties, this juurnal, Vol. 4, Theorem 1. 
of dimension s over $K$, the induced valuation $v^{\prime}$ of $k(x)$ is of dimension not smaller than s over $k$.

Proof. Let $R$ denote the valuation ring of $v$ in $K(x), A$ its valuation ideal, and let $R^{\prime}$ denote the valuation ring of $v^{\prime}$ in $k(x), A^{\prime}$ its valuation ideal. As $v$ is of dimension $s$, there are $s$ elements $y_{1}, \ldots, y_{s}$ in $R$ which are algebraically independent $\bmod A$ over $K$. Let $K_{0}$ be a finite extension field of $k$, such that all $y_{1}, \ldots, y_{s}$ belong to $K_{0}(x)$. Then $v$ induces the valuation of $K_{0}(x)$ of dimension $\geqslant s$ over $K_{0}$. Therefore we may assume that $K$ is a finite extension field of $k$.

$$
\begin{aligned}
& R / A \supseteqq R^{\prime} / A^{\prime} \supseteqq k, \\
& R / A \supseteqq \quad K \geqq k .
\end{aligned}
$$

Let the dimension of $K$ over $k$ be $t$. Then $R / A$ is of dimension $s+t$ over $k$. On the other hand the dimension of $R / A$ over $R^{\prime} / A^{\prime}$ is $\leqq t$. For, if $Z_{1}, \ldots, Z_{t+1}$ are $t+1$ elements in $R$, then as the dimension of $K(x)$ over $k(x)$ is $t$, there is an algebraic relation among them:

$$
\sum a_{r_{1}} \ldots r_{t+1} Z_{1}^{r_{1}} \ldots Z_{t+1}^{r_{t+1}}=0
$$

where all $a_{r_{1}} \ldots r_{r^{+1}}$ belong to $k(x)$. We may assume that all $a_{r_{1}} \ldots r_{t+1}$ belong to $R^{\prime}$ and there is an element among them which does not belong to $A^{\prime}$. Considering this relation $\bmod A$, we see that the dimension of $R / A$ over $R^{\prime} / A^{\prime}$ is $\leqq t$. Therefore the dimension of $R^{\prime} / A^{\prime}$ over $k$ is $\supseteq s$.

From this lemma we see that $\mathfrak{P}$ induces in $k(\mathbf{P})$ the valuation $\mathfrak{p}$ of dimension at least $n-1$. As $\omega(\mathbf{P})$ is the differential form of the first kind belonging to the extension $k(\mathbf{P})$ of $k, \omega(\mathbf{P})$ is finite at $\mathfrak{p}$; hence $\omega(\mathbf{P})$ is ninite at $\mathfrak{P}$. This completes the proof of Theorem 1 .

$\S 2$. We prove the following

TheOREM 2. Let $\mathrm{U}^{n}$ be a projective model without singular point and let $\omega$ be a differetial form on $\mathbf{U}^{n}$, defined over $k$. Let $\mathbf{U}^{\prime n-1}$ be the generic hyperplane section of $\mathrm{U}^{n}$ (over $k$ ) on which $\omega$ induces the differential form $\omega^{\prime}$ of the first kind. Then $\omega$ is of the first kind."

Proof. Let $\mathbf{U}^{\prime}$ be the intersection of $\mathbf{U}^{n}$ and a hyperplane $\mathbf{H}$ defined by a homogeneous equation

$$
\sum_{i=0}^{N} u_{i} X_{i}=0
$$

in $\mathbf{P}^{v}$. where $u_{0}, u_{1}, \ldots, u_{v}$ are algebraically independent over $k$. Let $\mathbf{W}^{n-1}$ be a subvariety of $\mathbf{U}^{n}$ which is algebraic over $k$ and let $\mathbf{W}^{\prime n-2}$ be a component of $\mathbf{W} \cap \mathbf{H}$, which is contained in $\mathbf{U}^{\prime}$.

4) This theorem has been proved also by S. Koizumi. 
Without loss of generality we may assume that $\mathrm{W}^{n-1}$ has a representative $\mathbf{W}_{0}^{n-1}$. Put $K=k\left(u_{0}, \ldots, u_{*}\right)$ and let $P=(x)$ be a generic point of $U_{v}^{\prime}$ over $\bar{K}$ and $Q$ a generic point of $\mathbf{W}_{u}^{\prime}$ over $\bar{K}$. Then $P$ is also a generic point of $U_{0}$ over $k$ and $Q$ is a generic point of $W_{1}$ over $k$. Further we may assume that $x_{1}$, $\ldots, x_{n}$ is a set of uniformizing parameters for $U_{11}$ at $Q$. Then it is easily seen that $x_{2}, \ldots, x_{n}$ is a set of uniformizing parameters for $U_{0}^{\prime}$ at $Q$. For simpicity we assume that $\omega$ is a simple differential form. We may treat the case of the differential form of the higher degrees analogously.

Let $\omega$ be defined by $\omega(P)=\sum_{i=1}^{n} a_{i} d x_{i}, a_{i} \in k(P)$. Then $\omega^{\prime}$ is defined over $\bar{K}$ by $\omega^{\prime}(P)=\sum_{i=1}^{n} a_{i} d x_{i}$, where $\sum_{i=1}^{n} a_{i} d x_{i}$ is considered as the differential form belonging to the extension $\bar{K}(P)$ of $\bar{K}$. Now since

$$
\begin{aligned}
& u_{0}+u_{1} x_{1}+\ldots+u_{N} x_{N}=0, \\
& \sum_{j=1}^{N} u_{j} d x_{j}=0, \text { i.e. } \\
& -u_{1} d x_{1}=\sum_{k=2}^{N} u_{k} d x_{k} .
\end{aligned}
$$

If we put $d x_{k}=\sum_{i=1}^{n} b_{k i} d x_{i}, b_{k i} \in k(P), k=n+1, \ldots N$, we get

$$
\begin{aligned}
& -u_{1} d x_{1}=\sum_{i=2}^{n} u_{i} d x_{i}+\sum_{k=n+1}^{N} u_{k} d x_{k} \\
& =\sum_{i=2}^{n}\left(u_{i}+\sum_{k=n+1}^{x} u_{k} b_{k i}\right) d x_{i}+\sum_{k=n-1}^{N} u_{k} b_{k 1} d x_{1} \text {. } \\
& -d x_{1}=\sum_{i=2}^{n} \frac{\left(u_{i}+\sum_{k=n+1}^{+} u_{k} b_{k i}\right)}{\left(u_{1}+\sum_{k=n+1}^{+} u_{k} b_{k 1}\right)} d x_{i} \\
& \omega^{\prime}(P)=\sum_{i=2}^{n}\left(a_{i}-a_{1} \frac{\left(u_{i}+\sum_{k=n+1}^{N} u_{k} b_{k i}\right)}{\left(u_{1}+\sum_{k=n+1}^{N} u_{k} b_{k 1}\right)}\right) d x_{i} .
\end{aligned}
$$

By the assumptions that $\omega^{\prime}$ is of the first kind and $x_{2}, \ldots, x_{n}$ form a set of uniformizing parameters,

$$
\begin{array}{r}
u_{i}+\sum_{k} u_{k} b_{k i} \\
A_{i}=a_{i}-a_{k} u_{k} b_{k 1}
\end{array}
$$

is in the specialization ring of $Q$ in $\bar{K}(P)$, therefore $A_{i}$ has a finite specialization over $P \rightarrow Q$ with respect to $\bar{K}$, and hence it has a finite specialization over $P \rightarrow Q$ with respect to $k\left(u_{1}, \ldots, u_{*}\right)$. Now as $P$ is a generic point of $U_{0}^{n}$ over $k\left(u_{1}\right.$, $\left.\ldots, u_{N}\right)$ and $Q$ is a generic point of $W_{0}^{n-1}$ over $\bar{k}\left(u_{1}, \ldots, u_{*}\right)$, either $a$ or $1 / a$ is in the specialization ring $\mathfrak{D}$ of $Q$ in $k\left(u_{1}, \ldots, u_{*}\right)(P)$, where $a$ is an arbicrary 
element in $k\left(u_{1}, \ldots, u_{N}\right)(P)$. Therefore $A_{i}$ must be in the specialization ring $\mathfrak{D}$; moreover since $1 /\left(u_{1}+\sum_{k=n+1}^{N} u_{k} b_{k 1}\right)$ is in $\mathfrak{D}$,

$$
A_{i} /\left(u_{1}+\sum_{k} u_{k} b_{k 1}\right)=a_{i} u_{1}+a_{1} u_{i}+\sum_{k=n+1}^{X} u_{k}\left(a_{i} b_{k 1}+a_{1} b_{k i}\right)
$$

is in $D$ for $i=2, \ldots, n$, where $a_{i}$ and $b_{k j}$ are in $k(P)$. As $u_{1}, \ldots, u_{v}$ are algebraically independent over $k(P), a_{i}$ and $a_{1}$ must belong to the specialization ring of $Q$ in $k(P) .^{5 ;}$ This shows that $\omega(P)$ is finite at $Q$. Since $\omega$ is finite at the generic point of every $(n-1)$-dimensional subvariety of $U, \omega$ is of the first kind. ${ }^{6}$

Mathematical Institute, Nagoya University

5) See A. Weil's book, Foundations of Algebraic Geometry, Prop. 8 in Chapter IV.

6) See Prop. 4 of Koizumi's paper loc. cit. 2). 